Discussion

\title{
Response to the discussion on "Reconstruction of the envelope of non- Gaussian structural responses with principal static wind loads by N. Blaise, T. Canor and V. Denoël”
}

\author{
Nicolas Blaise $^{\mathrm{a}, *}$, Thomas Canor ${ }^{\mathrm{b}}$, Vincent Denoël ${ }^{\mathrm{a}}$ \\ a Department of Architecture, Geology, Environment and Construction, University of Liège, Liège, Belgium \\ b F.R.S.-FNRS, National Fund for Scientific Research, University of Liège, Liège, Belgium
}

\section{Introduction}

This closure is an item by item rebuttal of the points raised in the discussion by Kasperski (2017) regarding the applicability of the loadresponse correlation (LRC) method in a non-Gaussian framework. The LRC-based equivalent static wind loads (ESWLs) possess an attractive meaning in a Gaussian context: they correspond to the most probable internal forces corresponding to the occurrence of a structural response. In a non-Gaussian framework, the LRC-based ESWL, however, loses this probabilistic meaning of "most probable load pattern".

A general concept, the Conditional Expected Static Load, for which a probabilistic meaning remains in a non-Gaussian context is presented in Blaise et al. (2016). It is important to emphasize that the discusser does not call into question the formal concept of Conditional Expected Static Wind Load as a relevant extension of the LRC method in a nonGaussian framework.

\section{Load-response correlation method applicability}

The LRC method can be used out of the Gaussian context, where it loses its physical meaning. This suggests assessing the optimality of the LRC-based ESWLs to reconstruct the envelope in a non-Gaussian framework.

Indeed the example given in Blaise et al. (2016) shows that significant overestimations of the entire envelope may be produced by applying several LRC-based ESWLs, see Figures 13 and 14. Although quite small, overestimations are also observable in the example given in Kasperski (2017).

\section{Conditional sampling method}

The conditional sampling technique (CST) is correctly applied in Blaise et al. (2016). The CST-based ESWL does not require a scaling coefficient alpha if the standard sampling approach is used to define the envelope. If another method is used to define the envelope, the scaling factor alpha is not unity. Regardless of the definition of the envelope, CST-based ESWLs may produce overestimations of the envelope, i.e., the coefficients beta may not be unity.

\section{The bicubic model}

The bicubic model is an approach to obtain conditional expected static loads. The bicubic model is relevant since (i) it offers a closed form expression of the conditional expected loads and (ii) it is in-line with the widely known cubic translation model (Winterstein, 1988). This is not the only way to determine the conditional expected static load and the scientific community is free to contribute with alternatives.

It is evident that the statistical parameters used for the bicubic model are affected by the limited amount of data. A proper study of the sampling distribution of the parameters of the bicubic model goes largely beyond the scope of the paper.

Finally, the monotone limitation of the bicubic model is clearly stated in Blaise et al. (2016), see the bottom of page 67, left column.

\section{Determination of the envelope}

The envelope values in Blaise et al. (2016) are not based on peaks picked from 10 minutes intervals as claimed by Kasperski (2017). The envelope values in Blaise et al. (2016) are computed with non-Gaussian peak factors based on the works of Kareem and Zhao (1994), see end of Section 2.

For a given project, the chosen definition of the envelope is an important matter of concern. On the contrary, in a general scientific work, it is important to prove efficiency in various contexts, which was done in Blaise (2016). A commonly used envelope has been chosen in Blaise et al. (2016), for the purpose of giving an example. A reference period of 10 minutes and the mean extremes (with the Kareem-Zhao model) are selected. In compliance with the specifications of every new project, other representative periods and other definitions of the envelope are possible.

Results for a reference period of 1 hour are presented in Blaise

\footnotetext{
* Corresponding author.

E-mail address: n.blaise0@gmail.com (N. Blaise)
} 
(2016), where it is shown that this reference period has only a slight influence on the envelope reconstruction efficiency. In Blaise (2016), it is moreover illustrated that considering the reference period of 1 hour actually eases the envelope reconstruction. It is, therefore, a false statement that considering a reference period of 10 minutes leads to a "less-challenging example of application." as stated in Kasperski (2017).

Finally, other definitions can be obviously considered for the envelope. The codification norm in force provides the extreme value to consider for the design, for instance in terms of non-exceedance probabilities.

\section{Envelope reconstruction problem: with or without the average field}

The average wind load straightforwardly provides the mean values of structural responses. The reconstruction illustrated in Blaise et al. (2016) intentionally focused on the zero-mean envelope of structural responses aiming at the fairest error estimate. The error estimate (that includes the mean), as proposed by the discusser would indeed result in substantially smaller errors.

It is true that it may not be necessary to reproduce all structural responses. The cost function defined in equation (43) in Blaise et al. (2016) allows selecting them by "engineering judgment" if desired. The primary purpose of the methodology is, however, to be general and not confined to simple frames and arches whose structural design is properly captured with two or three major easily identified responses.

\section{Conclusion}

To advance the state-of-the-art, the meaningful definition of a conditional expected static wind load is a solid concept for the developments of further methods to compute static wind loads in a non-Gaussian context. Besides, the bicubic model for joint nonGaussian random variables is relevant for our purpose since it offers closed-form solutions of conditional values. Moreover, it can be interpreted as a formal two-dimensional extension of the well-established cubic translation model from Winterstein (1988). Future enhancements would be particularly necessary for processes with skewness and excess coefficients outside the monotone limitation or processes with bi-modal distributions, for instance.

\section{References}

Blaise, N. 2016. Principal static wind loads within a rigorous methodology to the envelope reconstruction problem (Doctoral dissertation). Université de Liège, Liège, Belgium. http://orbi.ulg.ac.be/bitstream/2268/187599/1/Thesis.pdf

Blaise, N., Canor, T., Denoël, V., 2016. Reconstruction of the envelope of non-Gaussian structural responses with principal static wind loads. J. Wind Eng. Ind. Aerodyn. $149,59-76$.

Kareem, A., Zhao, J., 1994. Analysis of non-Gaussian surge response of tension leg platforms under wind loads. J. Offshore Mech. Arct. Eng. 116 (3), 137-144.

Kasperski, M., 2017. Discussion on: "Reconstruction of the envelope of non-Gaussian structural responses with principal static wind loads" authored by Blaise, N., T. Canor and V. Denoël, (2016).

Winterstein, S.R., 1988. Nonlinear vibration models for extremes and fatigue. J. Eng. Mech. 114 (10), 1772-1790. 